\title{
Valoración del estudiantado universitario sobre las competencias matemáticas para la resolución de problemas
}

\author{
University student's valuation of mathematical competences for problem solving
}

William Oswaldo Flores López ${ }^{1}$

Elena Auzmendi Escribano ${ }^{2}$

\section{Resumen}

Esta investigación ha evaluado las competencias matemáticas para la resolución de problemas del estudiantado universitario. Fue un estudio cuantitativo desde un diseño descriptivo donde se suministró un cuestionario sobre las competencias matemáticas para la resolución de problema a 876 hombres y mujeres estudiantes de la Universidad de las Regiones Autónomas de la Costa Caribe Nicaragüense (URACCAN). Los resultados confirmaron que las competencias matemáticas para la resolución de problemas se asocian con los procesos matemáticos de pensar y razonar, argumentar y justificar, comunicar, representar, modelar, plantear y resolver problemas; se ha observado que tanto los hombres y las mujeres tienen la misma capacidad para resolver problemas matemáticos; las diferencias estadísticamente significativas están en relación con la variable étnica a favor del pueblo miskitu, seguidamente de los creoles y mestizos, y con menores diferencias los mayangnas. Se concluye a través de este estudio, que las competencias matemáticas para la resolución de problemas pueden contribuir en el aprendizaje del estudiantado y en la práctica docente del profesorado en la enseñanza universitaria.

Palabras clave: Competencia matemática; resolución de problema; procesos matemáticos; género; etnia.

\section{Abstract}

This research has evaluated the mathematical competences for problem solving of university students. It was a quantitative study from a descriptive design where a questionnaire was supplied on the mathematical competences for problem solving to 876 male and female students of the University of the Autonomous Regions of the Nicaraguan Caribbean Coast (URACCAN). The results confirmed that mathematical competences for solving problems are associated with the mathematical processes

\footnotetext{
1 Doctor en Educación. Profesor Investigador de la Universidad de las Regiones Autónomas de la Costa Caribe Nicaragüense. Correo: william.flores@uraccan.edu.ni ID de ORCID: http://orcid.org/oooo-ooo2-1016-1620

2 Doctora en Psicología. Vicerrectora de Ordenación Académica, Innovación Docente y Calidad de la Universidad de Deusto. Correo: elena.auzmendi@deusto.es ID de ORCID: https://orcid.org/oooo-0oo1-6637-2630
}

Recibido: 05/02/2018 Aprobado: 30/04/2018 


\section{EDUCACIÓN}

of thinking and reasoning, arguing and justifying, communicating, representing, modeling, posing and solving problems; It has been observed that both men and women have the same ability to solve mathematical problems; the statistically significant differences are related to the ethnic variable in favor of the Miskitu people, followed by the Creoles and mestizos, and with minor differences the Mayangnas. It is concluded through this study, that the mathematical competences for the resolution of problems can contribute in the students learning and in the teaching practice of professors in higher education.

Keywords: Mathematic Competence; problem solving; mathematic processes; gender; ethnicity.

\section{Introducción}

Esta investigación tiene el propósito de evaluar las competencias matemáticas para la resolución de problemas del estudiantado universitario. Se parte del hecho de que, la resolución de un problema matemático y su desarrollo curricular en el aula, son en la actualidad un tema significativo dentro de los planteamientos del sistema educativo nicaragüense. A partir de esto, es que los más importantes avances se centran en el carácter transversal de la resolución de problemas como contenido matemático y el carácter concreto que adquiere su desarrollo curricular en la sala de clases.

Es importante señalar que en la Universidad de las Regiones Autónomas de la Costa Caribe Nicaragüense se imparte el curso de Matemática Básica, una asignatura que está dirigida al estudiantado de nuevo ingreso, y se plantea como la base para continuar estudios específicos de cada grado académico. Aproximadamente un 90\% de las unidades temáticas que se estudian están planteadas en el programa de matemática de educación secundaria y bachillerato. De manera que, en el curso, muchos de los temas son abordados como un complemento de la educación matemática recibida en la secundaria. Sin embargo, las estadísticas de los exámenes de admisión y los cursos propedéuticos que realizan las universidades muestran que un alto porcentaje de estudiantes nicaragüense acceden a estudios universitarios con carencias en sus habilidades matemáticas, que impiden el aprendizaje de algunas de las nuevas materias en la universidad y abordar con éxito la resolución de problemas.

Es por ello, que en este artículo se presentan los hallazgos obtenidos relacionados con la competencia matemática del estudiantado universitario para la resolución de problemas. Las competencias matemáticas del estudiantado revelan su experiencia de formación matemática, la cual constituye evidencias de construcciones adecuadas de su manera de entender a las matemáticas. 


\section{Revisión de literatura}

La competencia matemática se define como la capacidad del individuo para formular, emplear e interpretar las matemáticas en distintos contextos. Incluye el razonamiento matemático y la utilización de conceptos, procedimientos, datos y herramientas matemáticas para describir, explicar y predecir fenómenos. Ayuda a los individuos a reconocer el papel que las matemáticas desempeñan en el mundo y a emitir los juicios y las decisiones bien fundadas que los ciudadanos constructivos, comprometidos y reflexión necesitan (PISA, 2015, p. 74). Así mismo, Belmonte, Llinares, Ruiz, Rubio y Rivilla (2003), asocian la competencia matemática con el desarrollo de cinco dimensiones de manera integrada: Comprensión conceptual, destreza procedimental, comunicar, explicar y argumentar matemáticamente, pensamiento estratégico y actitudes positivas hacia la capacidad de aprender matemáticas.

Por efectos de la evaluación de la competencia matemáticas, puede analizarse en función de tres aspectos interrelacionados: El contenido matemático que se busca para utilizarlo en las preguntas de la evaluación; los procesos matemáticos, que describen lo que hacen los individuos para relacionar el contexto del problema con las matemáticas y de ese modo resolver y la capacidad que subyacen a esos procesos; y los contextos en los que se insertan las preguntas de evaluación. En referencia con el contenido, nos basamos en las ideas de Frege (1996) sobre sentido y referencia, quien sostiene que los diferentes significados de un concepto matemático vienen dados por las estructuras conceptuales que se insertan, por los sistemas de símbolos que lo representan, y por los objetos y fenómenos de los que surge y que le dan sentido. Rico (2006) adecua la terna de Frege, signo-sentido-referencia, para caracterizar el significado de un concepto matemático en contexto educativo. Esta reflexión sobre contenidos, corresponde al estudio curricular, el significado de un concepto matemático educativo que se establece mediante la terna Representación-Fenómeno-Definición.

Rico (1997), dice que resulta difícil asumir que hay multiplicidad de significados para un mismo concepto matemático, ya que hay diversos sistemas de signos para su representación e, igualmente, hay diversos objetos (fenómenos) que se requieren y dan distintos sentidos a un mismo concepto. Por eso es factible entender que hay pluralidad de significados para un mismo concepto matemático. Determinar los significados más relevantes de cada concepto es uno de los retos de la enseñanza-aprendizaje de las matemáticas, es decir, una tarea de investigación del profesor de matemáticas para trasmitir a los estudiantes los significados culturales y científicos de las matemáticas. "Entender diferentes significados de un mismo concepto proporciona su comprensión" (Rico, 2006, p. 285). En este contexto, las ideas fundamentales adoptada por OECD (2005), que satisfacen las condiciones de respetar el desarrollo histórico, cubrir el dominio y contribuir a la reflexión de las líneas principales del currículo escolar, son: Cantidad, espacio y forma, cambios y relaciones, incertidumbre. 
En relación con los procesos matemáticos, en PISA (2015) se centra en el proceso de matematización, el cual consiste en traducir los problemas desde el mundo real al matemático, por lo que se sustenta en varios subprocesos entre ellos: Pensar y razonar, argumentar y justificar, comunicar, representar, modelar, representar y plantear y resolver problemas (NCTM, 2000; TIMSS, 2003; OECD, 2005). Los procesos matemáticos formalizan la matematización en la resolución de problemas matemáticos. Según Pajares, Sanz y Rico (2004) su utilidad se concreta en establecer capacidades y habilidades específicas que ayudan a modular los objetivos, a establecer tareas escolares y caracterizar las propuestas de trabajo y las evaluaciones. Las capacidades y habilidades puestas en juego determinan que una persona es competente en matemáticas, son expresión de su conocimiento matemático. Los objetivos de aprendizaje de manera concreta son las habilidades que se necesitan para un determinado tema y en un determinado momento.

Finalmente, los contextos en los que se insertan las preguntas de evaluación, según Goñi (2008) se organizan en campos estructurantes como personal-familiar, social-comunitario, profesional-especializado, científico-tecnológico: Personal-familiar, relacionado con las actividades diarias, ya que el estudiantado debe activar sus conocimientos matemáticos para interpretar los aspectos relevantes de situaciones cotidianas; social-comunitario, se refiere a situaciones donde el estudiando debe relacionar diversos elementos del entorno social o entorno comunitario, para evaluar qué aspectos del mismo tienen consecuencias relevantes; profesional-especializado, referido a situaciones que surgen en la universidad o trabajo relacionada con su especialización con problemas que requieren una solución matemática; científicotecnológico, incluye contenidos más abstractos como la comprensión de procesos tecnológicos o la explicación de problemas matemáticos. Esta categoría abarca también situaciones del cálculo diferencial e integral abstracto que pueden surgir en las clases, y que requieren explicitar los elementos matemáticos del problema para situarlo en contexto más amplio.

\section{Materiales y métodos}

\section{Propósito y objetivos del estudio}

Esta investigación ha partido del hecho de que un objetivo en la enseñanza y aprendizaje de las matemáticas es que el estudiantado desarrolle la competencia matemática para la resolución de problemas. Además, que la resolución de cualquier problema matemático lleva asociada una situación afectiva para el sujeto implicado, quien pone en juego no solamente prácticas operativas y discursivas para dar una respuesta al problema, sino también moviliza creencias, actitudes, emociones y valores que condicionan, en mayor o menor grado y diferente sentido, la respuesta cognitiva requerida (Godino, 2013). Partiendo de estos planteamientos, el objetivo de esta investigación se sintetiza en evaluar las competencias matemáticas para la resolución de problemas del 
estudiantado universitario, tomando en consideración el género del estudiantado y la etnia de los mismos. Por tales razones, se propone los objetivos específicos siguientes:

- Determinar la competencia matemática de resolución de problemas del estudiantado universitario.

- Comprobar si existen diferencias significativas entre género en relación con los procesos matemáticos de resolución de problemas.

- Estudiar si existen diferencias significativas entre etnia en relación con los procesos matemáticos de resolución de problemas.

\section{Método de investigación}

El enfoque de esta investigación es cuantitativo. Este paradigma ha guiado el tratamiento de los datos a través de la categorización y descripción de las propiedades, características y perfiles de las personas, grupos, comunidades, procesos y objetos que se han sometido a análisis (Hernández, Fernández, \& Baptista, 2010). El diseño de esta investigación es diseño descriptivo ya que tiene como objetivo indagar la incidencia de las modalidades o niveles de una o más de una población (Bisquerra, 2012).

\section{Descripción de los participantes}

El estudio se llevó a cabo con una muestra de 876 estudiantes universitarios de los grupos étnicos miskitu (pueblos originarios), mayangna (pueblos originarios), creole (afrodescendientes) y mestizos de la Universidad de las Regiones Autónomas de la Costa Caribe Nicaragüense, pertenecientes a comunidades de la Costa Caribe de Nicaragua.

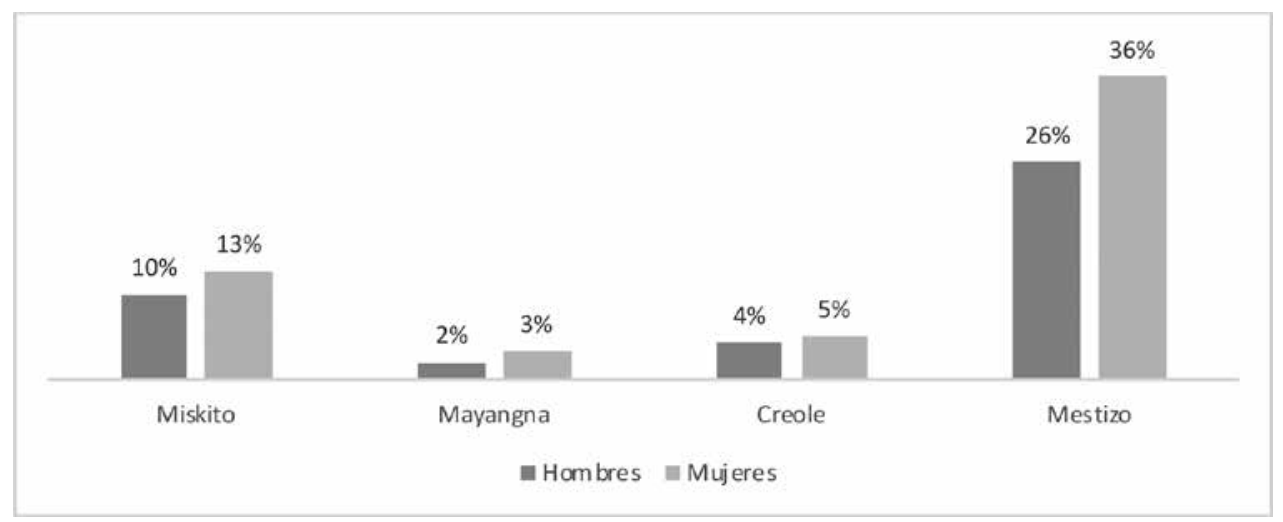

Figura 7: Distribución de los participantes en función de etnia y género

$\mathrm{El}$ 58\% (504) de los participantes son mujeres, frente al 42\% (372) que son hombres. Respecto a su edad, la media se sitúa en 18 años, encontrándose al $84 \%$ de los participantes en el intervalo de edad entre 18-21 años. En referencia a la variable etnia: el 23\% son indígenas miskitu; el 5\% indígenas mayangna; el 9\% creole (afrodescendientes); y 


\section{EDUCACIÓN}

el $62 \%$ mestizos. Otra característica de los participantes es que todos son estudiantes de nuevo ingreso y han recibido el curso de matemática para la vida al ingresar a la universidad.

Para concluir, indicar que el tipo de muestreo que hemos utilizado ha sido el muestro no probabilístico causal o accidental, que es aquel en el cual el investigador selecciona directa e intencionalmente la muestra, debido fundamentalmente a que tiene fácil acceso a la misma y es representativa de la población (Gil, Rodríguez y García, 1995; Albert, 2006; Sabariego, 2004).

\section{Instrumento de recogida de datos}

El instrumento para la recopilación de la información fue el cuestionario de competencias matemáticas para la resolución de problemas matemáticos de Flores y Auzmendi (2016). El instrumento está constituido por 32 ítems, aglutinados en 6 competencias o procesos de matematización: Pensar y razonar, argumentar y justificar, comunicar, modelar, representar, plantear y resolver problemas.

Cuadro 5: Procesos matemáticos en la resolución de problemas

\begin{tabular}{|l|l|}
\hline \multicolumn{1}{|c|}{ Proceso Matemático } & \multicolumn{1}{c|}{ Descripción } \\
\hline Pensar y razonar: & $\begin{array}{l}\text { Pensar en matemática es construir conocimiento matemático en situaciones } \\
\text { donde tenga sentido, experimentar, intuir, relacionar conceptos y abstraer. } \\
\text { Razonar en matemáticas es realizar deducciones e inducciones, particularizar } \\
\text { y generalizar; argumentar las decisiones, así como los procesos y las técnicas } \\
\text { (Alsina, 2004). }\end{array}$ \\
\hline Argumentar: & $\begin{array}{l}\text { Conjunto de habilidades, conocimientos y actitudes dirigidas a la explicación } \\
\text { de determinados procesos, entre las capacidades que incluye está la manera } \\
\text { en que explica la solución de un problema, cómo se justifica la solución del } \\
\text { mismo y cómo se demuestra su solución (Tobón, 2007). }\end{array}$ \\
\hline Comunicar: & $\begin{array}{l}\text { Capacidad de expresarse tanto de manera oral, como escrita con referencia a } \\
\text { asuntos con contenidos matemáticos, abarcar las capacidades de la forma en } \\
\text { que se expresa y representa información matemática y la manera en que se } \\
\text { interpreta dicha información (Niss, 2002), ayuda a los estudiantes a organizar } \\
\text { y consolidar su pensamiento matemático, debido a que representa una de las } \\
\text { claves para la profundización de la comprensión matemática (Silbey, 2003). }\end{array}$ \\
\hline $\begin{array}{l}\text { Construcción de } \\
\text { modelos: }\end{array}$ & $\begin{array}{l}\text { Se entiende así las matemáticas como un conjunto de ideas y formas de actuar } \\
\text { que conllevan a obtener modelos e identificar relaciones y estructuras (Castro, } \\
\text { Molina, Gutiérrez, Martínez y Escorial, 2012). }\end{array}$ \\
\hline Representar: & $\begin{array}{l}\text { Habilidad para utilizar y relacionar los símbolos y las formas de expresión y ra- } \\
\text { zonamiento matemáticos (Castro, et al, 2012). }\end{array}$ \\
\hline problemas: & $\begin{array}{l}\text { Habilidad práctica para resolver lo desconocido, comprende las capacidades } \\
\text { de identificar, plantear y resolver diferentes tipos de problemas matemáticos } \\
\text { utilizando una variedad de métodos (Niss, 2002). }\end{array}$ \\
\hline
\end{tabular}


Para garantizar la calidad de la medida, se aplicó un estudio psicométrico al instrumento para comprobar los valores de validez y fiabilidad del mismo. Se calculó el valor de consistencia interna del total de los ítems del cuestionario, obteniendo un valor alfa de Cronbach del 92\% de confiabilidad. Respecto a la validez, se realizó un análisis de componentes principales (ACP). La prueba Kaiser-Meyer-Olkin arrojó una puntuación de o,822. Por su parte, la prueba de esfericidad de Bartlett también ofreció resultados que indicaban que el análisis era pertinente (Chi-cuadrado=7467,046; g.l.=496; $\mathrm{p}<0,000)$. Además, se calculó el determinante de la matriz de correlaciones, cuyo valor fue prácticamente o $(\mathrm{D}=5.41 \mathrm{E}-10)$.

\section{Procedimientos de administración y enfoque ético}

La administración del instrumento se realizó por parte del autor durante el curso académico 2015-2016. Tenía un carácter anónimo y fue complementado por los sujetos participantes en presencia del profesorado. Con anterioridad a la toma de los datos, se obtuvo tanto el consentimiento previo libre e informado del estudiantado, profesorado, así como la autorización de las autoridades universitarias.

\section{Resultados}

\section{Competencias matemáticas para la resolución de problemas}

A partir de los distintos procesos de la competencia matemática para la resolución de problema, se calculó la puntuación que obtendría cada participante de la muestra en cada uno de los procesos matemáticos (pensar y razonar, argumentar y justificar, comunicar, modelar, representar, plantear y resolver problemas). El resultado se analiza desde una perspectiva descriptiva, indicando los niveles de competencias del estudiantado participante en relación con los temas de interés de esta investigación. Se analizó la puntuación total del cuestionario, así como las distintas agrupaciones, según los procesos matemáticos que se asocian al instrumento que evalúan la competencia matemática.

Los resultados muestran que la puntuación total media del cuestionario es 19,27 puntos ( $\mathrm{SD}=6,65 ;$ Error=0,22). A la vista de estos resultados se puede afirmar que la competencia matemática del estudiantado universitario es positiva (rango posible entre 1 y 32 puntos). Así mismo, al no alcanzarse valores extremos en la puntuación, la gran mayoría se concentra en una competencia positiva alrededor de 23 puntos (moda), competencia matemática que podríamos considerar moderada con tendencia alta.

Con una media entre 0.59 y 0.81 puntos se presenta el proceso de pensar y razonar, y el estudiantado puede realizar tareas que involucren subprocesos como: Responder a cuestiones en contextos muy conocidos; responder a cuestiones en contextos muy poco familiares; responder a cuestiones complejas en multitud de contextos; $y$, formar 


\section{EDUCACIÓN}

y relacionar conceptos. Las dificultades del estudiantado en este proceso matemático es utilizar una representación bidimensional dada para contar o calcular elementos de un objeto $(M=0,59)$. Así como utilizar los datos relevantes de la descripción de una situación compleja y realizar los cálculos correspondientes $(M=0,65)$. Véase el cuadro 2.

Cuadro 6: Descriptores del proceso de pensar y razonar

\begin{tabular}{|l|l|}
\hline \multicolumn{1}{|c|}{ Tareas específicas que el estudiantado debe ser capaz de realizar } & M \\
\hline Sustituir correctamente los números para aplicar un algoritmo numérico o fórmula algebraica sencilla & 0,68 \\
\hline Realizar cálculos sencillos que implican relaciones entre dos variables conocidas. & 0,73 \\
\hline Utilizar una representación bidimensional dada para contar o calcular elemento de un objeto. & 0,59 \\
\hline Llevar a cabo cálculos explícitamente descritos que comprenden los procesos secuenciales. & 0,81 \\
\hline $\begin{array}{l}\text { Utilizar los datos relevantes de la descripción de una situación compleja y realizar los cálculos corres- } \\
\text { pondientes. }\end{array}$ & 0,65 \\
\hline $\begin{array}{l}\text { Realizar cálculos sencillos que implican relaciones entre una variable conocida. Situación algebraica } \\
\text { en contexto de la propiedad conmutativa. }\end{array}$ & 0,78 \\
\hline $\begin{array}{l}\text { Reconocer e identificar en una situación problemática la presencia de algo desconocido que puede } \\
\text { ser determinado considerando restricciones del problema. }\end{array}$ & 0,73 \\
\hline
\end{tabular}

En el proceso de argumentar y justificar las puntuaciones oscilan entre o.61 y o.64 puntos, los estudiantes fueron capaces de elaborar argumentos basados en sus acciones, formular razonamientos desarrollados y elaborar argumentos desde su propia reflexión. Sin embargo, el estudiantado presentó la mayor dificultad al realizar una reflexión sobre las relaciones entre una fórmula algebraica y los datos subyacentes $(M=0,61)$, así como interpretar y explicar información matemática compleja en el contexto de una situación real desconocida $(M=0,64)$. Dichos conflictos influyen en la manera en que el estudiantado explica la solución de un problema y, también, en cómo justifica y demuestra su solución. Obsérvese la cuadro 3.

Cuadro 7: Descriptores del proceso de argumentar y justificar

\begin{tabular}{|l|l|}
\hline \multicolumn{1}{|c|}{ Tareas específicas que el estudiantado debe ser capaz de realizar } & M \\
\hline Interpretar y conocer representaciones complejas para resolver un problema práctico. & 0,64 \\
\hline $\begin{array}{l}\text { Reflexionar, interpretar y explicar información matemática compleja en el contexto de } \\
\text { una situación real desconocido. }\end{array}$ & 0,61 \\
\hline
\end{tabular}

El proceso de comunicar oscila entre 0.36 y 0.81 puntos. El estudiantado universitario tiene dificultades en comunicar resultados, las razones de este hecho pueden encontrarse en que los estudiantes realizaron tareas de los subprocesos de describir resultados obtenidos, realizar explicaciones sencillas y comunicar conclusiones con precisión. Las dificultades se producen porque el estudiantado no realizó tareas como aplicar algoritmos rutinarios para resolver problemas geométricos $(M=0,36)$; 
interpretar, aplicar, manejar y utilizar fórmulas verbales y fórmulas lineales que representen relaciones del mundo real $(M=0,48)$; y simbolizar las cantidades desconocidas identificadas en una situación específica y utilizarlas para plantear ecuaciones $(M=0,49)$. Véase la cuadro 4 .

Cuadro 8: Descriptor del proceso de comunicar

\begin{tabular}{|l|l|}
\hline \multicolumn{1}{|c|}{ Tareas específicas que el estudiantado debe ser capaz de realizar } & M \\
\hline Sustituir la variable por el valor o los valores que hacen de la ecuación un enunciado verdadero. & 0.72 \\
\hline $\begin{array}{l}\text { Interpretar, aplicar, manejar y utilizar fórmulas verbales y fórmulas lineales que represente relacio- } \\
\text { nes del mundo real. }\end{array}$ & 0.48 \\
\hline Sustituir correctamente los números para explicar las relaciones entre dos variables desconocidos. & 0.63 \\
\hline Interpretar las relaciones entre dos variables a través de la sustitución de un número conocido. & 0.81 \\
\hline Aplicar algoritmos rutinarios para resolver problemas geométricos. & 0.36 \\
\hline $\begin{array}{l}\text { Simbolizar las cantidades desconocidas identificadas en una situación específica y utilizarlas para } \\
\text { plantear ecuaciones. }\end{array}$ & 0.49 \\
\hline
\end{tabular}

En el proceso de modelar los estudiantes alcanzaron puntuaciones entre o.61 y o.73. Estos resultados muestran que, los estudiantes respondieron correctamente a tareas que relacionan los subprocesos de usar modelos explícitos en situaciones concretas y, desarrollar y usar modelos en múltiples situaciones. La problemática subyacente al proceso modelar consiste en que, el estudiantado reflexione sobre la relación entre una fórmula algebraica y los datos subyacentes $(M=0,61)$, por consiguiente, necesita desarrollar y usar modelos en múltiples situaciones. Obsérvese el cuadro 5 .

\section{Cuadro 9: Descriptores del proceso de modelar}

\begin{tabular}{|l|c|}
\hline \multicolumn{1}{|c|}{ Tareas específicas que el estudiantado debe ser capaz de realizar } & M \\
\hline $\begin{array}{l}\text { Utilizar representaciones múltiples para resolver un problema práctico, es decir, inter- } \\
\text { pretar y comprender significados. }\end{array}$ & 0.73 \\
\hline Reflexionar sobre la relación entre una fórmula algebraica y los datos subyacentes. & 0.61 \\
\hline
\end{tabular}

En el proceso de plantear y resolver problemas, el estudiantado obtuvo puntuaciones entre 0.42 y 0.63 puntos. Los estudiantes son capaces de realizar subprocesos como: Resolver problemas con datos sencillos, seleccionar y aplicar estrategias sencillas, seleccionar, comparar, evaluar estrategias y generalizar resultados de problemas. Las dificultades en el proceso de plantear y resolver problemas que se acentúan más con la necesidad de utilizar los datos relevantes de la descripción de una situación compleja y realizar los cálculos correspondientes $(M=0,42)$; sustituir la variable por el valor o los valores que hacen de la ecuación un enunciado verdadero, en un contexto aditivo y multiplicativo $(M=0,46)$; y usar comprensivamente el álgebra para resolver problemas; capacidad de mejorar expresiones algebraicas para traducir una situación del mundo real $(M=0,57)$. En definitiva, el estudiantado enfrenta el reto de concebir y elaborar una estrategia que transforme la información dada para llegar a una conclusión. Incluso 


\section{EDUCACIÓN}

con el uso de una estrategia para encontrar una solución exhaustiva o una conclusión generalizadora. Véase el cuadro 6.

Cuadro 10: Descriptores del proceso de plantear y resolver problemas

Tareas específicas que el estudiantado debe ser capaz de realizar

Sustituir la variable por el valor o los valores que hacen de la ecuación un enunciado verdadero, en un contexto aditivo y multiplicativo.

Utilizar los datos relevantes de la descripción de una situación compleja y realizar los cálculos correspondientes.

Usar comprensivamente el álgebra para resolver problemas; capacidad de mejorar expresiones algebraicas para traducir una situación del mundo real.

Aplicar la comprensión geométrica profunda para trabajar con patrones complejos y generalizados

El proceso de representar es necesario para que el estudiantado diseñe una representación que refleje los aspectos clave de una situación compleja, las puntuaciones oscilan entre 0.38 y 0.70 puntos. En este sentido, los estudiantes poseen las habilidades de representar que se refleja en los subprocesos siguientes: Leer datos directamente de tablas y figuras; usar un único tipo de representación; conocer y usar diferentes sistemas de representación; vincular diferentes sistemas de representación incluyendo el simbólico; y, relacionar y traducir con fluidez diferentes sistemas de representación.

Cuadro 11: Descriptores del proceso de representar

\begin{tabular}{|l|l|}
\hline \multicolumn{1}{|c|}{ Tareas específicas que el estudiantado debe ser capaz de realizar } & \multicolumn{1}{|c|}{ M } \\
\hline $\begin{array}{l}\text { Determinar la cantidad desconocida que aparece en el problema realizando operaciones algebraicas y } \\
\text { aritméticas. }\end{array}$ & 0.43 \\
\hline $\begin{array}{l}\text { Identificar los cálculos simples necesarios para resolver un problema sencillo en un contexto multipli- } \\
\text { cativo. }\end{array}$ & 0.45 \\
\hline Utilizar términos y definiciones técnicas básicas y aplicar conceptos geométricos básicos. & 0.46 \\
\hline Realizar cálculos sencillos que incluyan las operaciones aditivas de los números reales. & 0.67 \\
\hline Realizar cálculos sencillos que implican operaciones multiplicativas. & 0.70 \\
\hline $\begin{array}{l}\text { Interpretar y aplicar formulas verbales y manejar y utilizar formulas lineales que represente relaciones } \\
\text { del mundo real. }\end{array}$ & 0.69 \\
\hline $\begin{array}{l}\text { Interpretar la variable simbólica como la representación de una entidad general, indeterminada, que } \\
\text { puede asumir cualquier valor. }\end{array}$ & 0.38 \\
\hline Aplicar algoritmos rutinarios para relacionar diferentes representaciones de objetos. & 0.70 \\
\hline $\begin{array}{l}\text { Interpretar el lenguaje algebraico en los cuales se oculte algún algoritmo sencillo y aplicar dicho algo- } \\
\text { ritmo. }\end{array}$ & 0.58 \\
\hline $\begin{array}{l}\text { Analizar un modelo matemático dado que implique una fórmula compleja, es decir, deducir reglas y } \\
\text { métodos generales en secuencias de familias de problemas. }\end{array}$ & 0.48 \\
\hline
\end{tabular}




\begin{tabular}{|l|c|}
\hline \multicolumn{1}{|c|}{ Tareas específicas que el estudiantado debe ser capaz de realizar } & M \\
\hline $\begin{array}{l}\text { Manipular (simplificar y desarrollar) la variable simbólica en situaciones algebraicas en contexto aditivo } \\
\text { y multiplicativo. }\end{array}$ & 0.59 \\
\hline
\end{tabular}

Las dificultades en el proceso de representar se sitúan en tareas específicas como:

- Interpretar la variable simbólica como la representación de una entidad general, indeterminada, que puede asumir cualquier valor $(M=0.38)$;

- Determinar la cantidad desconocida que aparece en el problema realizando operaciones algebraicas y aritméticas $(M=0.43)$.

- Identificar los cálculos simples necesarios para resolver un problema sencillo en contexto multiplicativo $(M=0.45)$.

- Utilizar términos y definiciones técnicas básicas y aplicar conceptos geométricos básicos $(M=0.46)$.

- Analizar un modelo matemático dado que implique una formula compleja, es decir, deducir reglas y métodos generales en secuencias de familias de problemas $(M=0.48)$.

- Interpretar el lenguaje algebraico en los cuales se oculte algún algoritmo sencillo y aplicar dicho algoritmo $(M=0.58)$.

- Manipular (simplificar y desarrollar) la variable simbólica en situaciones algebraicas en contexto aditivo y multiplicativo $(M=0.59)$.

\section{Género y etnia en la competencia matemática para la resolución de problemas}

El cuadro 8, muestra que las puntuaciones promedias que obtienen hombres y mujeres en los diferentes procesos matemáticos y por ende en la competencia matemática para la resolución de problemas.

Cuadro 12: Puntuaciones promedias en función de la variable género

\begin{tabular}{|l|l|l|l|l|}
\hline \multicolumn{1}{|c|}{ Procesos Matemáticos } & \multicolumn{1}{|c|}{ Género } & \multicolumn{1}{c|}{ Media } & \multicolumn{1}{c|}{ Desviación estándar } & \multicolumn{1}{c|}{ Media de error estándar } \\
\hline \multirow{3}{*}{ Pensar y Razonar } & Hombres & 4.981 & 1.719 & 0.089 \\
\cline { 2 - 5 } & Mujeres & 4.986 & 1.7615 & 0.078 \\
\hline \multirow{3}{*}{ Argumentar y Justificar } & Hombres & 1.239 & 0.790 & 0.040 \\
\cline { 2 - 5 } & Mujeres & 1.254 & 0,769 & 0.034 \\
\hline \multirow{2}{*}{ Comunicar } & Hombres & 3.395 & 1.465 & 0.075 \\
\cline { 2 - 5 } & Mujeres & 3.551 & 1.446 & 0.064 \\
\hline
\end{tabular}


EDUCACIÓN

\begin{tabular}{|l|l|l|l|l|}
\hline \multicolumn{1}{|c|}{ Procesos Matemáticos } & \multicolumn{1}{|c|}{ Género } & \multicolumn{1}{c|}{ Media } & \multicolumn{1}{c|}{ Desviación estándar } & Media de error estándar \\
\hline \multirow{2}{*}{ Modelar } & Hombres & 1.317 & 0.774 & 0.040 \\
\cline { 2 - 5 } & Mujeres & 1.353 & 0.736 & 0.032 \\
\hline \multirow{2}{*}{$\begin{array}{l}\text { Plantear y resolver proble- } \\
\text { mas }\end{array}$} & Hombres & 2.064 & 1.191 & 0.061 \\
\cline { 2 - 5 } & Mujeres & 2.087 & 1.235 & 0.055 \\
\hline \multirow{2}{*}{ Representar } & Hombres & 6.024 & 2.610 & 0.135 \\
\cline { 2 - 5 } & Mujeres & 6.236 & 2.521 & 0.112 \\
\hline Competencia Matemática & Hombres & 19.022 & 6.718 & 0.348 \\
\hline & Mujeres & 19.468 & 6.605 & 0.294 \\
\hline
\end{tabular}

Se realizó un análisis de las puntuaciones medias en los procesos matemáticos y de la competencia matemática en función de la variable género. La prueba t-student para muestras independientes confirma que en los procesos matemáticos: el valor tstudent en pensar y razonar es $(p=0.967)$; el valor $t$-student en argumentar y justificar es $(p=0.782)$; el valor $\mathrm{t}$-student en comunicar es $(p=0.116)$; el valor $\mathrm{t}$-student en modelar es $(p=0.485)$; el valor $t$-student en plantear y resolver problemas es $(p=0.783)$; el valor t-student en representar es $(p=0.226)$; y el valor t-student en la puntuación global, es decir, en la competencia matemática es $(p=0.327)$, por consiguientes hombres y mujeres poseen iguales competencias matemáticas para la resolución de problemas.

También, se aplicó un análisis de varianza (ANOVA) en función de la variable etnia, encontrando que existen diferencias significativas en los procesos matemáticos: pensar y razonar es $(f=0.000)$; argumentar y justificar es $(f=0.000)$; comunicar es $(f=0.001)$; modelar es $(f=0.002)$; plantear y resolver problemas es $(f=0.000)$; y representar es $(f=0.001)$. Así mismo, en la puntuación global, es decir, en la competencia matemática para la resolución de problemas se encontraron diferencias significativas $(f=0.00 o)$. Por lo tanto, las etnias miskitus, mayangnas, creoles y mestizos poseen diferentes grados de competencias matemáticas para la resolución de problemas. Las comparaciones medias HSD-Tukey a nivel de cada proceso matemático dio como resultado que: la etnia miskitu y mestizo utilizan más el proceso de pensar y razonar que la etnia mayangna y creole; las etnias miskitu, creole y mestizo argumentan y justifican mejor sus resoluciones de problemas que la etnia mayangna; la etnias creole, miskitu y mestizos comunican mejor resultados que la etnia mayangna; las etnias miskitu, creole y mestizo modelan mejor situaciones del mundo real al mundo de las matemáticas que la etnia mayangna; las etnias creole, miskitu y mestizos plantean y resuelven problemas mejor que la etnia mayangna; y finalmente, las etnias creole, mestizo y miskitu representan las situaciones complejas mejor que la etnia mayangna. 
Cuadro 13: Comparaciones múltiples Prueba Post Hoc HSD-Tukey

\begin{tabular}{|c|c|c|c|c|c|c|}
\hline \multirow{2}{*}{ (I) Etnia } & \multirow{2}{*}{ (J) Etnia } & \multirow{2}{*}{$\begin{array}{c}\text { Diferencia } \\
\text { de medias (I-J) }\end{array}$} & \multirow{2}{*}{$\begin{array}{l}\text { Error } \\
\text { estándar }\end{array}$} & \multirow{2}{*}{ Sig. } & \multicolumn{2}{|c|}{ Intervalo de confianza al $95 \%$} \\
\hline & & & & & Límite inferior & Límite superior \\
\hline \multirow{3}{*}{ Miskito } & Mayangna & 5.092 & 1.064 & 0.000 & 2.351 & 7.833 \\
\hline & Creole & 0.162 & 0.857 & 0.998 & -2.044 & 2.368 \\
\hline & Mestizo & 0.880 & 0.541 & 0.365 & -0.514 & 2.275 \\
\hline \multirow{3}{*}{ Mayangna } & Miskito & -5.092 & 1.064 & 0.000 & -7.833 & -2.351 \\
\hline & Creole & -4.930 & 1.200 & 0.000 & -8.020 & -1.840 \\
\hline & Mestizo & -4.212 & 0.999 & 0.000 & -6.785 & -1.38 \\
\hline \multirow{3}{*}{ Creole } & Miskito & -0.162 & 0.857 & 0.998 & -2.368 & 2.044 \\
\hline & Mayangna & 4.930 & 1.200 & 0.000 & 1.840 & 8.020 \\
\hline & Mestizo & 0.718 & 0.774 & 0.790 & -1.276 & 2.712 \\
\hline \multirow{3}{*}{ Mestizo } & Miskito & -0.880 & 0.541 & 0.365 & -2.275 & 0.514 \\
\hline & Mayangna & 4.212 & 0.999 & 0.000 & 1.638 & 6.785 \\
\hline & Creole & -0.718 & 0.774 & 0.790 & -2.712 & 1.276 \\
\hline
\end{tabular}

A nivel global, se puede observar a través de los resultados de comparaciones medias múltiples del cuadro 9, que la etnia con mejor competencia matemática para la resolución de problemas, es decir, con mayor puntuación global, es la etnia miskitu (pertenecientes a un pueblo originario), seguidamente de la etnia creole (perteneciente a un grupo afrodescendiente) y la etnia mestizo; por el contrario, la etnia mayangna (perteneciente a un pueblo originario) tiene menor puntuación global, por consiguiente, una menor competencia matemática para la resolución de problemas.

\section{Conclusiones}

Esta investigación ha evaluado las competencias matemáticas para la resolución de problemas del estudiantado universitario y su relación con la variable género y etnia. Resulta relevante determinar la competencia matemática para la resolución de problemas, porque constituye una información valiosa tanto para la gestión, diseño, planificación e implementación de las metodologías de enseñanza por parte del profesorado, como para el desarrollo de los procesos de aprendizaje a generar en el estudiantado, quienes van a seguir interactuando con dicha disciplina no sólo en el ámbito académico, sino también en el personal y profesional a lo largo de toda la vida. Desde esta perspectiva, se encontró que la competencia matemática para la resolución de problemas del estudiantado universitario es positiva con una puntuación global de 19.27 puntos, dicha competencia matemática se resume de la manera siguiente:

La competencia matemática se asocia a la resolución de problemas en contextos personal-familiar, social-comunitario, profesional-especializado, científico-tecnológico: 


\section{EDUCACIÓN}

personal-familiar, concordando con las ideas de Arreguín, Alfaro y Ramírez (2012) que la competencia matemática para resolver problemas se vincula con su entorno sociocultural y acordes con sus objetivos, posibilidades e intereses a través del trabajo en equipo; generando oportunidades para utilizar diversos recursos como conocimientos disciplinarios e interdisciplinarios; involucrándose en actividades de investigación, movilizando conocimientos, saberes y prácticas; y aplicando capacidades o procesos como pensar y razonar, argumentar y justificar, comunicar, modelar, representar, plantear y resolver problemas. Además, estos resultados se relacionan con las ideas de Jiménez y De León (2017), en el sentido que los procesos o habilidades matemáticas se desarrollan a tempranas edades y son utilizadas a lo largo de todo el aprendizaje de las matemáticas.

En este sentido, el estudiantado, es capaz de pensar y razonar, esto es, construir conocimiento matemático en situaciones donde tenga sentido, experimentar, intuir, relacionar conceptos y abstraer. Aunque, el estudiantado no se aproximó a razonar matemáticamente, es decir, a realizar deducciones e inducciones que ayudaran a particularizar, generalizar y argumentar las decisiones, así como los procesos y las técnicas. Son capaces de elaborar argumentos basados en sus acciones, o sea, concretar un conjunto de habilidades, conocimientos y actitudes dirigidas a la explicación de determinados procesos de resolución de problemas, relacionándose con el proceso argumentar y justificar, que según Tobón (2007) cuando los estudiantes explican, justifican y realizan demostraciones están dominando esta competencia matemática de argumentar.

Asimismo, en el proceso de comunicar en matemáticas, el estudiantado describe los resultados obtenidos por medio de la interpretación de las relaciones entre dos variables a través de la sustitución de un número conocido. Sin embargo, tienen que mejorar la capacidad de expresarse tanto de manera oral, como escrita con referencia a asuntos con contenidos matemáticos. Para ello, es necesario abarcar las capacidades de la forma en que se expresa y representa información matemática y la manera en que interprete dicha información (Niss, 2002), esto ayuda al estudiantado a organizar y consolidar su pensamiento matemático, debido a que representa una de las claves para la profundización de la comprensión matemática (Silbey, 2003).

En referencia con el proceso de modelar, el estudiantado usa modelos explícitos en situaciones concretas, es decir, utiliza representaciones múltiples para resolver un problema práctico, es decir, interpretar y comprender significados. Aunque, tiene dificultades en desarrollar y usar modelos en múltiples situaciones, es por ello, que el estudiantado tiene que entender las matemáticas como un conjunto de ideas y formas de actuar que llevan a obtener modelos e identificar relaciones y estructuras (Castro, et al., 2012). Sobre plantear y resolver problemas, el estudiantado es capaz de generalizar resultados de problemas; no obstante, tiene dificultades en seleccionar y aplicar estrategias sencillas y seleccionar, comparar y evaluar estrategias. Por lo tanto, 
es necesario cultivar en el estudiantado la habilidad para resolver lo desconocido, con el objetivo que les permita comprender, identificar, plantear y resolver distintas situaciones del mundo real al mundo de las matemáticas utilizando una variedad de métodos (Niss, 2002).

Sobre el proceso de representación, el estudiantado es capaz de conocer y usar diferentes sistemas de representación, aunque tiene dificultades en leer datos directamente de tablas y figuras. Según Castro et al. (2012), la exigencia de este proceso de representación para el estudiantado implica la habilidad para utilizar y relacionar los símbolos y las formas de expresión y razonamientos matemáticos (Castro et al., 2012). Esto le ayudaría a comprender y utilizar una representación no estándar que requiere una notable descodificación e interpretación; diseñar una representación que refleje los aspectos clave de una situación compleja y comparar y valorar distintas representaciones.

Partiendo de la importancia de los resultados de las evaluaciones comparativas internacionales afirman que existen diferencias de género en matemáticas, a favor de los hombres (PISA, 2015), se estudió si existen diferencias estadísticamente significativas entre hombres y mujeres en relación con los procesos matemáticos y la competencia matemática global, encontrando que hombres y mujeres, participantes en esta investigación, muestran las mismas capacidades de resolución de problemas, coincidiendo con el estudio de Marbán, Martín, Ortega y De la Torre (2013), en el sentido, que las mujeres tienen las mismas competencias matemáticas para la resolución de tareas matemáticas, igual que los hombres.

También, la competencia matemática se manifiesta como elemento relevante en la variable etnia, y específicamente en la etnia miskitu (pueblos originarios), lo que indica que el estudiantado miskitu podría tener mejor competencia matemática para la resolución de problemas, y a la vez, creer que los procesos matemáticos constituyen una herramienta para dar soluciones optimas de la manera correcta; sin embargo, la etnia creole y mestiza obtuvieron puntuaciones positivas en relación a los resultados obtenidos en el cuestionario. Es necesario mencionar, que los resultados obtenidos por las etnias miskitu, creole y mestizo, juega un papel importante las emociones del estudiantado universitario en el desarrollo de la competencia matemática, como afirma Ruvalcaba et al. (2014), las emociones ayudan a los estudiantes a resolver problemas con mayor eficacia.

No obstante, la etnia mayangna (pueblos originarios) posee dificultades en el desarrollo de la competencia matemática, ésto podría explicarse por el hecho de que el estudiantado indígena mayangna aprende conceptos, definiciones, propiedades y proceso de resolución de problemas matemáticos en un idioma distinto a su lengua materna; aunque el Estado de Nicaragua reconoce las diferencias lingüísticas del país, no invierte en la revitalización de las lenguas indígenas y en la creación de palabras 


\section{EDUCACIÓN}

técnico-científico para que el estudiantado proveniente de poblaciones indígenas puedan asimilar los conceptos matemáticos avanzados. En concreto, es necesario implementar currículos y procesos de formación sensibles a la diversidad cultural, lo que contribuirá a sus formas de aprender y hacer matemáticas del estudiantado procedente de grupos étnicos.

Es importante señalar, que las distribuciones de los participantes son desiguales, pero esto responde a las brechas de desigualdades sociales, acceso y éxito académico en los estudios universitarios de estudiantes provenientes de poblaciones indígenas y afrodescendientes de Nicaragua. Es claro, entonces, que hay una responsabilidad por parte del profesorado para desarrollar y cultivar la competencia matemática en el estudiantado proveniente de los pueblos indígenas, afrodescendientes y mestizos, es decir, establecer estrategias que permitan beneficiar la predisposición favorable hacia las matemáticas del estudiantado con prácticas didácticas de la vida cotidiana que ayuden a mejorar la calidad de los aprendizajes, favoreciendo el desarrollo de los procesos, los contextos de resolución de problemas (personal-familiar, socialcomunitario, profesional-especializado, científico-tecnológico: y personal-familia); y las actitudes, creencias y emociones del estudiantado hacia las matemáticas.

\section{Lista de referencias}

Albert, M. (2006). La investigación educativa. Claves teóricas. Madrid: MCGraw-Hill.

Alsina, A. (2004). Desarrollo de competencias matemáticas con recursos lúdicos-manipulativos. Madrid: Narcea.

Arreguín, L. E., Alfaro, J., \& Ramírez, M. S. (2012). Desarrollo de competencias matemáticas en secundaria usando la técnica de aprendizaje orientado en proyecto. Revista Iberoamericana sobre calidad, eficacia y cambio en educación, 10(4), 265-284.

Belmonte, J., Llinares, S., Ruiz, M., Rubio, F., \& Rivilla, A. (2003). Didácticas de las matemáticas para Primaria. En M. Chamorro, Didácticas de las matemáticas para Primaria (págs. 222-272). Madrid: Pearson Prentice Hall.

Bisquerra, R. (2012). Metodología de la investigación educativa. Madrid: Muralla.

Castro, C., Molina, E., Gutiérrez, M., Martínez, S., \& Escorial, B. (2012). Resolución de problemas para el desarrollo de la competencia matemática en educación infantil. Números: Revista de didáctica de las matemáticas, 80, 53-70.

Flores, W., O., \& Auzmendi, E. (2015). Resolución de problemas matemáticos: un cuestionario para su evaluación y comprensión. Ciencia e Interculturalidad, 16(1), 54-74. DOI: http://dx.doi.org/10.5377/rci.v16i1.2353 
Frege, G. (1996). Escritos filosóficos. Barcelona: Critica.

Gil, J., Rodríguez, G., \& García, E. (1995). Estadística básica aplicada a las Ciencias de la Educación. Sevilla: Kronos.

Godino, J. (2013). Indicadores de la idoneidad didáctica de procesos de enseñanza y aprendizaje de las matemáticas. Cuadernos de Investigación y Formación en Educación Matemática, 11, 111-132.

Goñi, J. (2008). Ideas Clave. El desarrollo de la competencia matemática. Barcelona: Graó.

Hernández, R., Fernández, C., \& Baptista, M. (2010). Metodología de la investigación. México: McGraw-Hill.

Jiménez, J., E., \& De León, S., del C. (2017). Análisis factorial confirmatorio del IPAM en escolares de tercer curso primaria. Revista Evaluar, 17(2), 81-96.

Marbán, J. M., Martín, M. C., Ortega, T., \& De la Torre, E. (2013). Perfil emocional matemático y competencias profesionales. Revista Electrónica Interuniversitaria de Formación del Profesorado, 16(1), 73-96. DOI: https://doi.org/10.6018/ reifop.16.1.179451

NCTM. (2000). Principles and standards for school mathematics. VA: NCTM.

Niss, M. (2002). Mathematical competencies and the learning of mathematics. Denmark: IMFUFA-Roskilde University.

OECD. (2005). Informe PISA 2003. Aprender para el mundo de mañana. Madrid: Santillana.

Pajares, R., Sanz, A., \& Rico, L. (2004). Aproximación a un modelo de evaluación: el proyecto PISA 2000. Madrid: Ministerio de Educación, Cultura y Deporte.

PISA. (2015). Marcos y pruebas de evaluación de PISA 2015: Ciencias, Matemáticas, Lectura y Competencia Financiera. París: OECD.

Rico, L. (1997). Organizadores del currículo de matemáticas. En L. Rico, La educación matemática en la enseñanza secundaria. Barcelona: Horsori.

Rico, L. (2006). Marco teórico de evaluación PISA sobre matemáticas y resolución de problemas. Revista de Educación Matemática, Extraordinario, 275-294. 


\section{EDUCACIÓN}

Ruvalcaba, N., Gallegos, J., Lorenzo, M., \& Borges, A. (2014). Propiedades Psicométricas de competencias socioemocionales para adolescentes (EQi-YV) en población mexicana. Revista Evaluar, 14(1), 1-14.

Sabariego, M. (2004). El proceso de investigación. En R. Bisquerra, Metodología de la investigación educativa (págs. 127-163). Madrid: La Muralla.

Silbey, R. (2003). Math out loud! Instructor, 112(7), 24-26.

TIMSS. (2003). Marcos teóricos y específicamente de evaluación TIMSS 2003. Madrid: Ministerio de Educación, Cultura y Deporte-INCE.

Tobón, S. (2007). Formación basada en competencias. Pensamiento complejo, diseño curricular y didáctica. Colombia: Ecoe Ediciones. 